\title{
ACCESO DE LOS CIUDADANOS A LOS DOCUMENTOS COMO TRANSPARENCIA DE LA GESTIÓN PÚBLICA
}

\author{
Vicent Giménez-Chornet
}

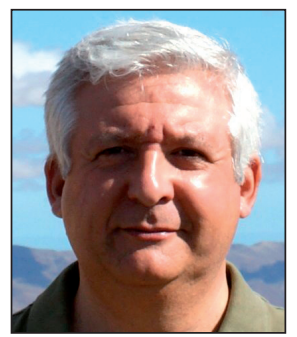

Vicent Giménez-Chornet es profesor titular de la Universitat Politècnica de València, con docencia en el Grado de gestión y administración pública y en el Máster de gestión cultural. Es presidente del Col/legi de Oficial de Bibliotecaris i Documentalistes de la Comunitat Valenciana, y miembro del Comité Técnico de Normalización de Documentación (AEN/CTN 50) de Aenor.

Universitat Politècnica de València Depto. de Comunicación Audiovisual, Documentación e Historia del Arte Camino de Vera, s/n. 46022 Valencia, España vigicho@har.upv.es http://www.vicentgimenez.net

\section{Resumen}

La transparencia en las administraciones públicas es posible si los ciudadanos tienen garantizado el acceso a los documentos. En el presente artículo proponemos una definición de la transparencia y analizamos la normativa legal española que trata el derecho de acceso. La transparencia es posible si también las instituciones tienen organizados sus archivos. Proponemos unos requisitos esenciales que deben cumplir la normativa legal y la gestión documental.

\section{Palabras clave}

Transparencia administrativa, Acceso a la información, Administración pública, Gestión de documentos.

Title: Public access to documents supports transparency in public administration

\section{Abstract}

Transparency in public administration is possible if citizens are guaranteed access to documents. In this paper we propose a definition of transparency and analyse the Spanish legislation that addresses the right of access to information. Transparency is also more readily achieved if institutions have organized their archives. We propose essential requirements that must meet the legal and records management standards.

\section{Keywords}

Administrative transparency, Access to information, Public administration, Records management.

Giménez-Chornet, Vicent. "Acceso de los ciudadanos a los documentos como transparencia de la gestión pública”. El profesional de la información, 2012, septiembre-octubre, v. 21, n. 5, pp. 504-508.

http://dx.doi.org/10.3145/epi.2012.sep.09

\section{Introducción}

La transparencia en la gestión pública no existe en valores absolutos. Es un objetivo a alcanzar y, en la medida en que los responsables públicos -gestores y políticos- la practiquen, la normativa legal lo posibilite y la gestión de los documentos sea una realidad, se habrá alcanzado un grado más o menos importante de eficacia en la transparencia de la gestión de los organismos públicos. La libertad de información es un requisito imprescindible para mejorar el acceso a la información pública, y para ello los gobiernos deben regular el marco de su aplicación. Los gobiernos saben que están obligados, en virtud de los tratados de derechos humanos que han ratificado, a implementar, promover y proteger estos derechos, incluido el derecho de acceso a la información en poder de organismos públicos (Mutula, 2006). La transparencia de los gobiernos hacia los ciudadanos se puede ejercer complementariamente por los medios de comunicación, siendo éstos un canal crucial para que los ciudadanos estén informados del funcionamiento del gobierno y de las maniobras en el sistema político, y como tal debería ser institucionalizado para que las comunicaciones de prensa gubernamentales fueran veraces, exactas y transparentes (Pearson; Patching, 2008).

La transparencia también puede ser ejercida por la empresa privada, no tanto por responsabilidad jurídica como por la repercusión que pueda tener en sus clientes. Hay estudios que demuestran el potencial que supone la transparencia de las empresas en relación con sus clientes, especialmente 
aquellas que desarrollan una política proambiental, apoyando un negocio sostenible (Vaccaro; Patiño-Echeverri, 2010). Igualmente se ha demostrado en algunos casos que la falta de transparencia, incluso la adopción de algunas estrategias fraudulentas en la gestión, puede dar beneficios a corto plazo, pero repercutir negativamente a largo plazo (Transparency, 2010).

La transparencia también está estrechamente relacionada con la práctica democrática de un estado. Se considera que la democracia representativa es un orden estable en el que ejercen políticos, asesores políticos y organismos de control, desarrollando y aplicando políticas, legitimadas si se realizan siempre en un marco democrático. En este sentido hay autores que consideran que se ha sobreestimado esta manera formal de legitimización democrática, porque opinan que los procesos políticos son procesos dinámicos, y no son siempre procesos lineales y racionales, siguiendo el modelo ideal de democracia representativa. En este contexto acciones como la transparencia y ofrecer la información correcta en el momento pertinente están relacionadas con la democracia representativa (Hanberger, 2003).

\section{Sin gestión de la información la transpa- rencia es extremadamente limitada}

Dependiendo de cómo entendemos el Estado y la administración pública, la transparencia y el derecho a la información se pueden ejercer en mayor o menor grado. Nosotros partimos de la concepción de que los representantes políticos y los gestores públicos, tanto el gobierno en todas sus manifestaciones, como las administraciones públicas, no son poseedores de los bienes que gestionan, sino que ejercen sus cargos en representación de los ciudadanos -que son quienes sufragan su coste-, y gestionan temporalmente -mientras dura su cargo administrativo o político- los bienes públicos. Simplificando, diferenciamos dos conceptos de la Administración y del Estado que tienen consecuencias diferentes sobre los ciudadanos: Por una parte tenemos el concepto del Estado social de derecho, en el cual la Administración realiza numerosas prestaciones para que los ciudadanos lleven una existencia decorosa, y el Estado colabora con los actores sociales -sindicatos y otros grupos organizados-, y por otra parte tenemos el New public management, que propone un nuevo modelo de gestión pública y de Administración, partiendo de la convicción de que el sector privado es más eficaz que el administrativo y al que debemos imitar, y propone un retroceso del Estado del bienestar insistiendo en la disminución del gasto público (Baena-del-Alcázar, 2005, pp. 37-44).

El modelo de Estado, de Administración y de la gestión de la información generada por éstos repercute en el alcance de la transparencia ejercida en una sociedad. La gestión de la información, especialmente la producida por los tres poderes -el Ejecutivo con la Administración, el Legislativo y el Judicial-, y generalmente depositada en los archivos de oficina o centrales, es esencial para garantizar el acceso de los ciudadanos a los documentos generados por sus re- presentantes políticos y por sus gestores -los funcionarios públicos-. Los medios tecnológicos -la administración electrónica- proporcionan una vía de acceso más eficaz, pero remarcamos que sin gestión de la información la transparencia es extremadamente limitada.

\section{Transparencia en la gestión pública}

Definimos la transparencia en la gestión pública como la posibilidad de que los ciudadanos consigan acceder a cualquier tipo de información, registrada en cualquier soporte, generada por las entidades en el desarrollo de sus funciones. Los límites puestos a la transparencia, justificados o interesados, legales o en la práctica, evidencian el nivel de cultura democrática de un país. Dejamos separada de esta definición la finalidad de la transparencia, por su amplitud de acepciones, de carácter político, social, económico o de otra índole.

La diferencia entre la transparencia en la gestión pública y la reutilización de la información, en la que los estados ponen a disposición de los ciudadanos los datos y la información que consideran convenientes, en el caso español el proyecto Aporta (Marcos-Martín; Soriano-Maldonado, 2011), radica en las posibilidades de acceso y en quién tiene la iniciativa. Los datos que se ofrecen en la reutilización de la información son accesibles, y la iniciativa es de los gobiernos -siguiendo las reivindicaciones del movimiento open data- que los ponen a disposición de los ciudadanos en diferentes sitios Web institucionales, cumpliéndose los dos requisitos de derecho y posibilidad de acceso. Sin embargo, las administraciones públicas guardan muchos más documentos e información que por su volumen aún es inalcanzable ponerlos a disposición de los ciudadanos vía online, y no ofrecen información sobre su catalogación en la Web. En este caso la iniciativa la promueve el ciudadano, que pide información, a la cual tiene derecho mientras no afecte a las restricciones legales de seguridad del estado, protección de la intimidad de las personas, u otras establecidas reglamentariamente, ya que todos los ciudadanos españoles "tienen derecho a acceder a los registros y a los documentos que, formando parte de un expediente, obren en los archivos administrativos, cualquiera que sea la forma de expresión, gráfica, sonora o en imagen o el tipo de soporte material en que figuren, siempre que tales expedientes correspondan a procedimientos terminados en la fecha de la solicitud" (España, Lrjappac, 1992, art. 37.1). Conseguir acceder a este inmenso volumen documental va más allá del movimiento open data. Tal volumen documental es el registro o testimonio de la actuación de los poderes públicos, y ponerlo a disposición de los ciudadanos requiere una normativa legal que lo ampare y unas medidas reales que garanticen su práctica. Ambos requisitos caracterizan la transparencia de los poderes públicos.

\section{Iniciativas legales para la transparencia en la gestión pública}

Los precedentes de la transparencia los podemos encontrar en la Declaración de los derechos del hombre y del ciudadano de 1789, durante la revolución francesa, que estipula claramente en su capítulo 15: "La sociedad tiene derecho a pedir a todos sus agentes cuentas de su administración”, pero 
que no tiene validez legal en España. La normativa europea es la primera que se elabora fuera de España -excepto la firma de tratados internacionales- que es vinculante para los españoles. En la Carta de los derechos fundamentales de la Unión Europea, cuya primera redacción es del año 2000, establece en su última actualización de 2010, art. 42, "todo ciudadano de la Unión y toda persona física o jurídica que resida o tenga su domicilio social en un Estado miembro tiene derecho a acceder a los documentos de las instituciones, órganos y organismos de la Unión, cualquiera que sea su soporte" (Unión Europea, Cdfue, 2010), sin establecer límites.

En España el Estado tiene un proyecto de ley de transparencia, y algunas comunidades autónomas se han anticipado emanando leyes. En Galicia, la Ley 4/2006, sobre transparencia, abarca solamente a la administración autonómica y establece un límite importante en su art. 4. 1, regulando que sólo tienen derecho a solicitar y obtener información sobre los aspectos de la actividad administrativa de las instituciones aquellas personas a las que afecten a sus derechos e intereses legítimos (Galicia, Ltbpap, 2006), impidiendo que cualquier ciudadano pueda consultar cualquier documento de las actuaciones administrativas. En este aspecto no hay ningún avance respecto al art. 37 de la Ley 30/1992, sino que marca un retroceso, ya que la ley estatal de 1992 establece de forma genérica en el art. 37.1 el completo derecho de los ciudadanos a acceder a los registros y a los documentos que obren en los archivos administrativos, sin la limitación referida a que deben afectar a derechos e intereses de las personas que lo soliciten -otra cuestión son los expedientes personales, que en la indicada Ley 30/1992 sí establece límites para proteger los datos personales- (España, Lrjappac, 1992).

La legislación española sobre la e-administración no se plantea el acceso de los ciudadanos a cualquier documento generado electrónicamente por las administraciones públicas, sino a sus trámites

Otras leyes o decretos han regulado en España el derecho de acceso a la información o a los documentos, con unos objetivos distintos a la transparencia, relacionados con el acceso a los servicios públicos en el ámbito de la e-administración. Este nuevo entorno tecnológico evidencia la necesidad de avanzar en materia de transparencia, dado que ofrece la posibilidad al ciudadano de interactuar electrónicamente con las administraciones públicas de forma inmediata (DeOcaña-Lacal, 2012). El Real decreto 1671/2009 acomete el desarrollar parcialmente la Ley 11/2007, de acceso electrónico de los ciudadanos a los servicios públicos, y se centra en facilitar los derechos de acceso a la información administrativa o la preservación de intereses de terceros, bajo las siguientes condiciones: en la medida que lo permite la tecnología, y garantizando que no resultan afectados otros bienes constitucionalmente protegidos, como la protección de datos. España no dispone de una ley de administración electrónica -que es un concepto más amplio- sino de leyes o normas para que los ciudadanos realicen trámites preestablecidos con las administraciones públicas -el acceso a los servicios públicos-, que es un concepto mucho más restringido. Por este motivo la legislación española sobre la e-administración no se plantea el acceso de los ciudadanos a cualquier documento generado electrónicamente por las administraciones públicas, sino a sus trámites. Excepcionalmente algunas administraciones públicas ponen en abierto diversos documentos generados electrónicamente, como los ayuntamientos en el caso de las actas de los plenos.

\section{El Real decreto 1708/2011 permite que las administraciones públicas continúen siendo poco transparentes}

Recientemente se han elaborado dos normas que tratan el acceso a los documentos administrativos. Una es el Real decreto $1708 / 2011$, por el que se establece el Sistema español de archivos y se regula el Sistema de archivos de la Administración General del Estado y de sus organismos públicos y su régimen de acceso (España, R. D. 1708/2011), y la otra, en el marco del Esquema nacional de interoperabilidad, la Resolución de 28 de junio de 2012, de la Secretaría de Estado de Administraciones Públicas, por la que se aprueba la Norma técnica de interoperabilidad de política de gestión de documentos electrónicos (España, Resolución, 28.06.2012). El real decreto pretende "facilitar el ejercicio del derecho de acceso de los ciudadanos a los archivos y documentos administrativos, mediante la clarificación de los criterios fijados en la muy diversa legislación aplicable y con la introducción de un procedimiento común, muy simplificado, de solicitud de acceso". El reto presenta una dificultad: el acceso a los documentos que contienen datos personales, cuando la mayor parte de los documentos administrativos contienen por necesidad datos personales, como por ejemplo una inspección de bibliotecas o un expediente de una contrata de un servicio. Se realiza una clasificación de acceso a estos documentos, pero no una valoración de qué datos personales son accesibles o no, remitiendo a la normativa de la Agencia de Protección de Datos. Esto permite que las administraciones públicas continúen siendo poco transparentes.

El único artículo novedoso del real decreto, el 28.4, es algo que ya se estaba practicando en algunos archivos históricos que contienen documentación reciente: "se concederá el acceso a documentos que contengan datos de carácter personal, sin necesidad de consentimiento de sus titulares, cuando se proceda previamente a la oportuna disociación de los datos de modo que se impida la identificación de las personas afectadas". En general dicho Real decreto no ha llenado el vacío legal existente (Desantes-Fernández, 2012). Esta normativa ampara la falta de transparencia por los poderes públicos, no sólo ante los ciudadanos, sino que es extensible a los representantes políticos en la oposición, como el caso de la negativa a explicar y mostrar documentación en una comisión de control de las Cortes Valencianas sobre los gastos de la contratación de RTVV por la venida del Papa Benedicto XVI (Comunidad Valenciana, Cortes valencianas, 2010). 
La resolución que aprueba la Norma técnica de interoperabilidad de política de gestión de documentos electrónicos, en su capítulo VI, sobre los procesos de gestión de documentos electrónicos, aborda la accesibilidad como una de las funciones de gestión de documentos en el ámbito concreto de la interoperabilidad entre administraciones, estableciendo como buena práctica el registro de la trazabilidad de las acciones que se realice en cada acceso. El Esquema nacional de interoperabilidad resuelve los requisitos para el intercambio y conservación de la información electrónica por parte de las administraciones públicas (Gobierno de España, 2012), pero no es su objetivo primordial la transparencia.

\section{Requisitos para la transparencia en la gestión de las administraciones públicas}

Los requisitos para alcanzar el mayor grado de transparencia de los actos realizados por las administraciones y gobiernos, en el marco de la definición antes propuesta, la subordinamos a dos áreas, la normativa y la gestión.

\subsection{Requisitos que debe cumplir la normativa}

- Establecer que toda persona tiene derecho a solicitar y recibir información y documentos de cualquier entidad de la administración y los poderes públicos, sin exigir causa para el ejercicio de este derecho.

- Regular el procedimiento para que el acceso sea eficaz.

- Establecer las excepciones al derecho de acceso a la información y los documentos. El nivel de excepciones determina el nivel de transparencia de un país. Generalmente las excepciones están relacionadas con la seguridad del estado -militar, económica, etc.-, los expedientes personales en el ámbito de la administración de justicia o de la salud, etc.

- Período de tiempo de vigencia de las excepciones desde que se originó el documento. España es uno de los países democráticos que más largos períodos tiene establecidos, dificultando cualquier tipo de investigación.

- Creación de un órgano completamente independiente de control y evaluación de la transparencia.

- Establecer que los nombres de las personas físicas o jurídicas que realicen actividades económicas con las administraciones públicas sean considerados información pública. Esta norma parte del principio de que los gestores públicos administran el erario público, de todos los ciudadanos, quienes tienen derecho a saber dónde, cómo y por qué se gasta el dinero.

\subsection{Requisitos que debe cumplir la gestión documental}

- Todas las administraciones públicas dispondrán de unidades de documentación y/o archivo que controlen su fondo para poder ofrecerlo a los ciudadanos.

- La producción documental debe estar organizada: clasificada y ordenada.

- La producción documental debe estar descrita.

- La producción documental debe estar indizada.

- La gestión documental debe estar automatizada.

- La gestión documental debe disponer de un sistema de recuperación de la información.

- Todo organismo productor de documentos debe disponer de un calendario de conservación, fruto del proceso de valoración documental, en el que se recoja el plazo de permanencia de los documentos en cada una de las fases su ciclo vital, para regularizar su selección, eliminación o conservación permanente.

\section{La legislación española no regula eficaz- mente y con claridad el derecho de acce- so a la información y los documentos}

\section{Conclusión}

Si consideramos la transparencia en la gestión pública como la posibilidad de que los ciudadanos consigan acceder a cualquier tipo de información, registrada en cualquier soporte, generada por las entidades en el desarrollo de sus funciones, la legislación española no regula eficazmente y con claridad el derecho de acceso a la información y los documentos, a causa de los vacíos normativos y de la acumulación de límites regulados en diversas disposiciones legales.

Para garantizar el ejercicio de la transparencia es esencial que los órganos productores de documentos cumplan un mínimo de requisitos que garantice la óptima gestión de sus fondos para dar servicio a las peticiones de información de los ciudadanos.

\section{Bibliografía}

Baena-del-Alcázar, Manuel. Manual de ciencia de la administración. Madrid: Síntesis, 2005, ISBN: 8497562755

Comunidad Valenciana, Cortes Valencianas, "Reunión 10; Punto 5. Comparecencia del director general de RTVV (Radio Televisión Valenciana) para explicar el proceso de contratación de las empresas que cubrieron para RTVV la visita a Valencia del Papa Benedicto XVI, solicitada por Grupo Parlamentario Socialista (RE 66.104)" [vídeo] Comisión de Control de la Actuación de la RTVV y Sociedades dependientes (09/12/2010).

http://anneo.cortsvalencianes.es/seneca/fp.asp?set= sesiones\&id=oai:seneca: sesiones $/ 161$ \& asset $=267 \&$ offset=00:57:18\&kw=Papa\#player_tab-267

De-Ocaña-Lacal, Daniel. "Ser o no ser: el archivero entre el derecho de acceso y la protección de la información". Tabula, 2012, n. 15, pp. 149-184.

Desantes-Fernández, Blanca. "El procedimiento de acceso desarrollado en el R. D. 1708/2011, de 18 de noviembre. Entre la realidad y el deseo". Tabula, 2012, n. 15, pp. 109-146.

España, Lrjappac. “Ley 30/1992, de 26 de noviembre, de Régimen jurídico de las administraciones públicas y del procedimiento administrativo común". Boletín oficial del Estado, n. 285, 27.11.1992, pp. 40300-40319.

España, R. D. 1671/2009. "Real decreto 1671/2009, de 6 de noviembre, por el que se desarrolla parcialmente la Ley 11/2007, de 22 de junio, de acceso electrónico de los ciudadanos a los servicios públicos". Boletín oficial del Estado, n. 278, 18.11.2009, pp. 97921-97948.

España, R. D. 1708/2011. "Real decreto 1708/2011, de 18 
de noviembre, por el que se establece el Sistema español de archivos y se regula el Sistema de archivos de la administración general del Estado y de sus organismos públicos y su régimen de acceso". Boletín oficial del Estado, n. 284, 25.11.2011, pp. 125573-125592.

España, Resolución. “Resolución de 28 de junio de 2012, de la Secretaría de Estado de Administraciones Públicas, por la que se aprueba la Norma técnica de interoperabilidad de política de gestión de documentos electrónicos". Boletín oficial del Estado, n. 178, 26.07.2012, pp. 53776-53780.

Galicia, Ltbpap. "Ley 4/2006, de 30 de junio, de transparencia y de buenas prácticas en la Administración pública gallega". Boletín oficial del Estado, n. 198, 19.08.2006, pp. 30724-30731.

Gobierno de España, Esquema nacional de interoperabilidad, 2012.

http://administracionelectronica.gob.es/?_nfpb=true\&_ pageLabel=PAE_PG_CTT_General\&langPae=es\&iniciativa= 145

Hanberger, Anders. "Democratic implications of public organizations". Public organization review: A global journal, 2003, n. 3, pp. 29-54.

http://dx.doi.org/10.1023/A:1023095927266

Marcos-Martín, Carlos; Soriano-Maldonado, Luis-Salvador. "Reutilización de la información del sector público y open data en el contexto español y europeo. Proyecto Aporta". El profesional de la información, mayo-junio 2011, v. 20, n. 3, pp. 291-297.

http://dx.doi.org/10.3145/epi.2011.may.07

Mutula, Stephen M. "Freedom of information in the SADC region: implications for development and human rights". $\mathrm{Li}$ brary review, 2006, v. 55, n. 7, pp. 440-449.

http://dx.doi.org/10.1108/00242530610682155

Pearson, Mark; Patching, Roger. "Government media relations: A 'spin' through the literature". Humanities \& social sciences papers, 2008, paper 228.

http://epublications.bond.edu.au/hss_pubs/228

"Transparency is the best policy: Avoiding corporate damage through appropriate crisis management". Strategic direction, 2010, v. 26, n. 10, pp. 22-24.

http://dx.doi.org/10.1108/02580541011080537

Unión Europea, Cdfue. "Carta de los derechos fundamentales de la Unión Europea (2010/c 83/02)". Diario oficial de la Unión Europea, 30.3.2010, pp. C 83/389-C 83/403.

http://eur-lex.europa.eu/LexUriServ/LexUriServ.do?uri=OJ: C:2010:083:0389:0403:ES:PDF

Vaccaro, Antonino; Patiño-Echeverri, Dalia. "Corporate transparency and green management". Journal of business ethics, 2010, n. 95, pp. 487-506.

http://dx.doi.org/10.1007/s10551-010-0435-z

\section{IraLIS es el registro de autoridades de nombres de autor del repositorio E-LIS}

\section{Antes de subir documentos a E-LIS debes iralizarte siguiendo los "Criterios de firma".}

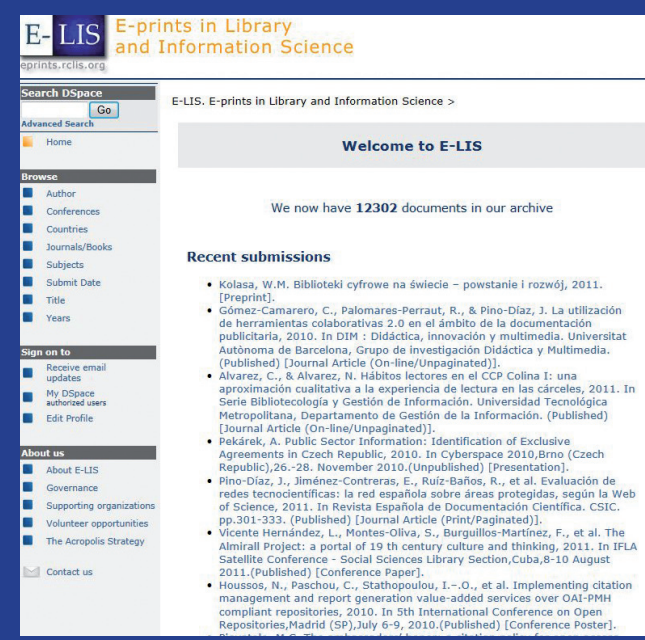

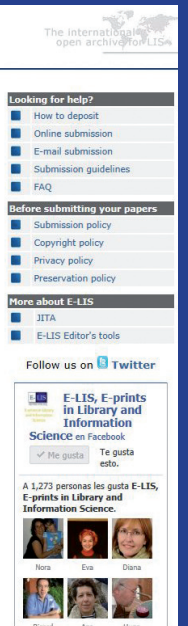

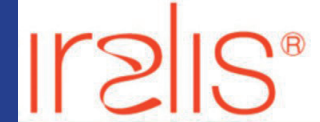

International Registry for Authors: Links to Identify Scientists

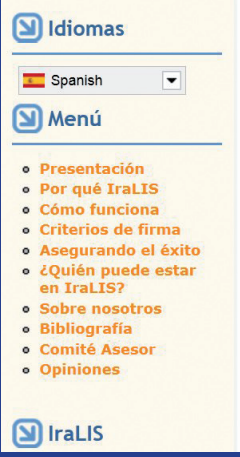

Presentación

IraLis (International Registry of Authors-Links to Identify Scientists) es un sistema de estandarización de las firmas de los autores científicos. Su objetivo es crear un registro de autoridades.

Trabaja en 5 líneas:

1. Concienciar a los autores científicos sobre la importancia de la firma para ser citados bibliografía a lo largo de su carrera profesional.

2. Redactar criterios de firma normalizada para a) ser indexado correctamente $y$ b) distinguirse de otros autores con nombres 